ARTICLE

https://doi.org/10.1057/s41599-019-0360-4

\title{
Trait specialization, innovation, and the evolution of culture in fluctuating environments
}

\author{
Dominik Deffner (1D $^{1 \star} \&$ Anne Kandler ${ }^{1}$
}

\begin{abstract}
Individuals often respond phenotypically to environmental challenges by innovating and adopting novel behavioral variants. Behavioral (or 'cultural') variants are defined here as alternative ways to solve adaptive problems, such as finding food or building shelter. In unpredictable environments, individuals must both be able to adapt to current conditions but also to cope with potential changes in these conditions, they must "hedge their evolutionary bets" against the variability of the environment. Here, we loosely apply this idea to the context of behavioral adaptation and develop an evolutionary model, where cultural variants differ in their level of generality, i.e. the range of environmental conditions in which they provide fitness benefits: generalist variants are characterized by large ranges, specialist variants by small ranges. We use a Moran model (with additional learning opportunities) and assume that each individual's propensity for innovation is genetically determined, while the characteristics of cultural variants can be modified through processes of individual and social learning. Our model demonstrates that flexibly adjusting the level of generality allows individuals to navigate the trade-off between fast and reliable initial adaptation and the potential for long-term improvements. In situations with many (social or individual) learning opportunities, no adjustment of the innovation rate, i.e. the propensity to learn individually, is required to adapt to changed environmental conditions: fast adaptation is guaranteed by solely adjusting the level of generality of the cultural variants. Few learning opportunities, however, require both processes, innovation and trait generality, to work hand in hand. To explore the effects of different modes of innovation, we contrast independent invention and modification and show that relying largely on modifications improves both short-term and long-term adaptation. Further, inaccuracies in social learning provide another source of variant variation that facilitates adaptation after an environmental change. However, unfaithful learning is detrimental to long-term levels of adaptation. Our results demonstrate that the characteristics of cultural variants themselves can play a major role in the adaptation process and influence the evolution of learning strategies.
\end{abstract}

\footnotetext{
${ }^{1}$ Department of Human Behavior, Ecology and Culture, Max Planck Institute for Evolutionary Anthropology, Leipzig, Germany. *email: dominik_deffner@eva.mpg.de
} 


\section{Introduction}

M

any of the challenges individuals face during their lifetime are fundamentally unpredictable. To be able to thrive in the face of such uncertainty, individuals often need to exhibit adaptations that respond to the diversity of environmental conditions they are likely to encounter in their lifetime (Slatkin, 1974; Starrfelt and Kokko, 2012). Adaptations often arise phenotypically by innovating ("innovation" here and in the following refers to any individual learning process that introduces new traits (Cavalli-Sforza and Feldman, 1981)) and socially transmitting novel behavioral variants through the population. Such social learning, i.e. learning that is facilitated by observation of, or interaction with, another individual or its products (Hoppitt and Laland, 2013; Heyes, 1994), is prevalent among many, taxonomically diverse, species, ranging from bumblebees and fish to cetaceans and chimpanzees (Galef and Laland, 2005; Laland, 2018), and is most prominently showcased in human cumulative cultural evolution (Mesoudi and Thornton, 2018). Innovation and social learning here refer to the functional outcome of learning processes that make individuals either acquire a new variant or match the behavioral variant of an interaction partner and do not imply any specific learning mechanisms. Populations of socially learning individuals can respond to changes in the environment by collectively building up and transmitting behavioral adaptations, that allow them to flexibly respond to variable environmental conditions. There is now a vast theoretical and empirical literature on the conditions that favor the evolution of social learning and the way individuals (should) combine individual and social information strategically in order to acquire locally adaptive behavior in variable environments (see e.g. Aoki and Feldman, 2014; Kendal et al., 2018, for reviews of the theoretical end empirical literature, respectively). In particular, theoretical modeling approaches have mainly focused on understanding which individual and social learning strategies are expected to have evolved in such environments. On a more proximate level, there is still debate about what exactly evolves in the evolution of social learning, with opinions ranging from changes in specialized learning brain circuitry to more subtle attentional or motivational differences between humans and animals less reliant on social learning (Leadbeater, 2015).

Characteristics of the cultural variants themselves, the target of learning, as well as the way they have been invented were largely ignored. But individuals may cope with environmental uncertainty not only by employing different learning strategies or changing innovation rates, but also by strategically adjusting certain characteristics of the cultural trait itself. In the past, models of cumulative culture and cultural complexity incorporated properties of cultural traits explicitly. These models usually treat cultural traits as symbol or number vectors that can vary in length which is taken to represent cultural complexity (Mesoudi, 2011; Fogarty et al., 2017). More elaborately, Kolodny et al. (2015) modeled the state of tool knowledge in a population as a combination of "main-axis tools", "toolkits" associated with them and "combinations" of these main-axis tools. Although elucidating, these kinds of models study trait complexity as their outcome measure and do not address how intrinsic properties of cultural traits themselves might facilitate adaptation in variable environments.

A modeling framework for addressing how trait characteristics may facilitate successful adaptation, has been put forward by Kandler and Laland (2013). They developed a mathematical model to study how different mixtures of individual and social learning strategies affect the frequencies of different variants of a cultural trait, and consequently the adaptation level of the population, in temporally and spatially changing environments. Cultural variants in this model are characterized by adaptation functions expressing the degree of benefit a variant conveys over a range of environmental settings. Some traits had narrow adaptation functions making them "specialist" solutions useful only in a very narrow range of environmental settings. More "generalist" variants, in contrast, had wider distributions, making them adapted to a broader range of conditions. They provided results about the interplay between social learning, in the form of payoffbiased and conformist learning, and individual learning, as well as the role of cultural diversity for an efficient adaptation process. However, they did not systematically investigate the role of such specialist and generalist variants in the adaptation process, nor did they look at the consequences these trait characteristics may have on the evolution of the propensity for individual learning, i.e. the rate of innovation.

In this paper, we build on the modeling framework by Kandler and Laland (2013) and develop an evolutionary model of behavioral adaptation in temporally variable environments that incorporates the possibility of specialized and generalist cultural variants. We aim to clarify the role such trait characteristics have for managing uncertainty in variable environments but also explore how innovation and trait specialization (or generality) act together in responding to changed environmental conditions. Rather than modeling a specific study system, we keep our model abstract enough to be applicable to a diverse range of contexts, where the propensity for social learning has a genetic basis and cultural variants can differ with respect to the range of environments in which they confer adaptive benefits. One could for example imagine different culturally learned food preferences in whales, songs in birds, or weapon technologies in humans.

Recently, several studies explored the consequences of different kinds of innovation, innovation rates, and "creativity" for cultural adaptation dynamics (and cultural complexity) (e.g. Kandler and Laland, 2009, 2013; Fogarty et al., 2015; Fogarty and Creanza, 2017; Fogarty, 2018). Lewis and Laland (2012), for instance, investigated the relative influence of transmission fidelity, novel invention, modification, and combination for the build-up of cumulative culture and found that while fidelity is key, modification and combination play a more important role than novel inventions. Addressing the impact of different innovation processes on the accumulation of cultural traits empirically, Miu et al. (2018) used data from 14 years of online programming competitions and explored the dynamics of cumulative culture in a system exhibiting real-world complexity. They report that, within each contest population, performance increased over time through a combination of many gradual modifications ("tweaks") and rare independent innovations ("leaps"). Following Lewis and Laland (2012) and Miu et al. (2018), we investigate how a combination of gradual modifications and rarer novel inventions influence adaptation dynamics. Lastly, we study the consequences of different degrees of copying error for short-term and long-term adaptation and, thereby, address the long-held assumption that high fidelity of transmission is a prerequisite for successful cultural adaptation and cumulative cultural evolution (Lewis and Laland, 2012; Heyes, 1993; Tennie et al., 2009).

\section{The model}

We consider a population of $N$ individuals which experience a temporally changing environment, described by $E(t)$. The environment can assume any value between -1 and 1 , whereby values closer together represent environmental conditions that are more similar in the dimension of interest. Each individual $j$ is characterized by its propensity to engage in social learning, denoted by $1-\xi_{j}$ with $\xi_{j} \in[0,1]$, and the cultural variant it has adopted at time $t$. 
Cultural variants. A cultural variant $i$ is defined by its adaptation function $a_{i}(E(t))$ which expresses the benefit the variant conveys to its carriers in the environmental state $E(t)$

$$
a_{i}(E(t))=a_{\max , i} \mathrm{e}^{-\frac{\left(E(t)-\mu_{i}\right)^{2}}{2 \sigma_{i}^{2}}} .
$$

The variable $\mu_{i}$ represents the environmental condition to which variant $i$ is optimally adapted, $\sigma_{i}$ the width of the adaptation function, which can be interpreted as the level of generality (the larger $\sigma_{i}$ the more general is variant $i$ ), and $a_{\max , i}$ its maximum adaptation level (see Fig. 1a).

To prevent the evolution of an "Ubervariant", which possesses high adaptation levels in a large range of environmental conditions, the maximum adaptation level of a variant, $a_{\max , i}$, decays exponentially with increasing $\sigma_{i}$. It holds $a_{\max , i}=e^{-10 \sigma_{i}}$. This expression implements a trade-off between specialist variants that convey high adaptation levels in a narrow range of environmental conditions and generalist variants that convey lower maximum adaptation levels but cover a broader range of environmental conditions (see Fig. 1a). Adaptation levels can vary between 0 and 1 with 1 describing optimal adaptation to the present conditions. Further, it holds $\mu_{i} \in[-1,1]$ and $\sigma_{i} \in(0,0.2]$. Summarizing, a cultural variant $i$ is characterized by the parameter pair $\left(\mu_{i}, \sigma_{i}\right)$ and the adaptation function (1).

Learning. At the beginning of each timestep $k$ individuals are randomly selected from the population to update their cultural variant through learning. As we are interested in how different genetic and cultural processes contribute to adaptation to changing environments, we included a parameter $k$ for the number of learning opportunities per timestep that effectively controls how fast cultural variants can evolve relative to genetic evolution. Each selected individual $j$ engages in innovation with probability $\xi_{j}$ and in social learning with probability $1-\xi_{j}$.

Innovation means that individuals acquire a new cultural variant through either novel invention or modification, i.e. the probability $\xi_{j}$ can also be interpreted as innovation rate of individual $j$. Novel inventions occur with probability $1-m$ and are defined by randomly drawing the $\mu$ - and $\sigma$-value of the new variant from the uniform distributions $\mathcal{U}(-1,1)$ and $\mathcal{U}(0,0.2)$, respectively, allowing individuals to acquire a cultural variant that is independent of their present variant (see e.g. Lewis and Laland, 2012). In contrast, modification occurs with probability $m$ and assumes that individuals do not invent a new variant from scratch

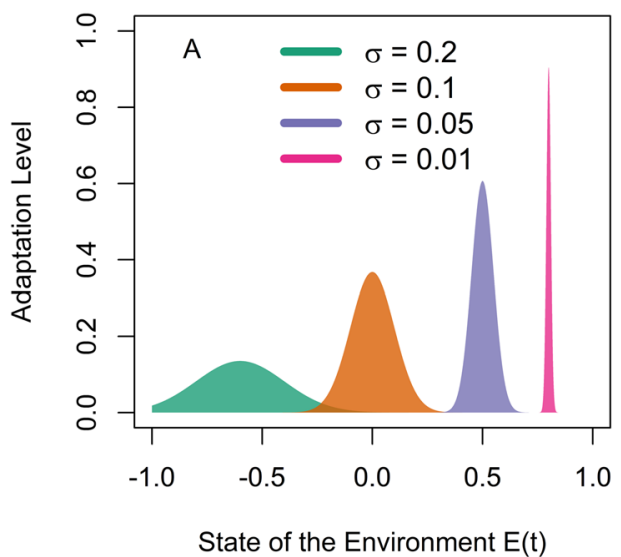

but modify the variant they have already adopted (see e.g. Lewis and Laland, 2012). In detail, an individual modifies its current variant characterized by $\left(\mu_{i}, \sigma_{i}\right)$ by adding a value drawn from $\mathcal{N}(0,0.1)$ to $\mu_{i}$ and a value drawn from $\mathcal{N}(0,0.01)$ to $\sigma_{i}$. If this process results in parameter values outside the allowed ranges for $\mu_{i}$ or $\sigma_{i}$, new values are drawn until the variant remains within the allowed boundaries. We note that our model does not include potential recombination processes between different cultural variants. This remains an interesting focus of future research. While many models (especially those on the adaptive value of culture (e.g. Rogers, 1988; Boyd and Richerson, 1988) assume that individual learners pay a cost to reliably acquire adaptive behavior through for example trial-and-error learning, we do not assume an additional learning cost, as individuals here just try out some new variant without exploring the environment (see also Fogarty and Creanza, 2017; Fogarty, 2018; Kolodny et al., 2015).

Social learning means that individuals randomly select three interaction partners from the population from whom they may copy a cultural variant. This stems from the assumption that individuals may not be able to interact with the whole population at the same time. However, over the course of multiple learning events individuals interact with different members of the population (letting social learners select a model from the whole population leads to very similar results; see Fig. A1 in the Appendix). If individuals possess a variant that conveys higher adaptation levels than the variants of all interaction partners, then they keep their own variant. If this is not the case, individuals choose among the interaction partners with a probability proportional to the relative adaptation level of their respective variants. Individuals acquire the variant of the selected model either faithfully, i.e. individuals are able to reliably adopt the observed behavior, or unfaithfully. In the case of unfaithful learning a value drawn from $\mathcal{N}\left(0, \sigma_{\text {error }}\right)$ with $\sigma_{\text {error }}=0.01$ or $\sigma_{\text {error }}=0.05$ is added to the $\mu$-value of the variant that is being copied and a value drawn from $\mathcal{N}(0,0.01)$ is added to the respective $\sigma$-value. Summarizing, social learners in our model follow a payoff-biased social learning strategy (Boyd and Richerson, 1988).

Birth-death process. Birth and death events, which occur after learning has taken place, were implemented through a Moran process (Moran, 1958). In each timestep, one individual is chosen for reproduction and one individual dies; thus ensuring that the population size remains constant over time. In more detail, an

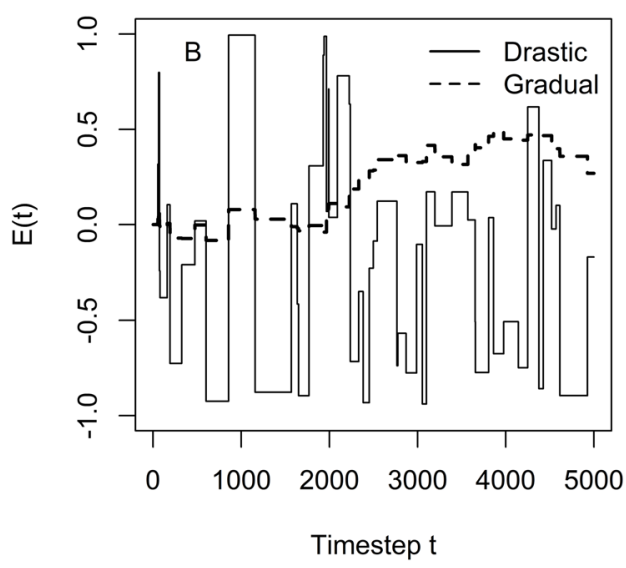

Fig. 1 a lllustrative example of four different adaptation functions with decreasing levels of generality (from left to right; indicated by smaller $\sigma$ ). Broader distributions cover wider ranges of environmental conditions but are accompanied by lower maximum adaptation levels. $\mathbf{b}$ Illustration of different modes of environmental change (with $\varepsilon=0.01$ ): solid line shows drastically fluctuating environment (new state drawn from $\mathcal{U}(-1,1)$ ), dashed line shows gradually fluctuating environment $\left(\sigma_{\varepsilon}=0.05\right)$. 
individual is chosen for reproduction with a probability proportional to its relative adaptation level determined by the adaptation function (1) of the cultural variant it has adopted.

The newborn inherits the $\xi$-value of its parent with probability $1-\mu_{\xi}$ and with probability $\mu_{\xi}$ a mutation occurs. In this case a new $\xi$-value is drawn from the uniform distribution $\mathcal{U}(0,1)$. As the precise process by which an individual's propensity to engage in social learning mutates is not known we also implemented a more gradual process, where the offspring value deviates slightly from the parent value: $\xi_{\text {offspring }}=\xi_{\text {parent }}+\delta$ with $\delta \sim \mathcal{N}\left(0, \sigma_{\xi}\right)$. Now the newborn, $j$, engages in vertical social learning, i.e. it copies the variant of its parent with probability $1-\xi_{j}$, or innovates through novel invention with probability $\xi_{j}$.

In contrast to the birth process, death occurs at random; one individual, which may also be the one that was chosen for reproduction, is randomly selected to die irrespective of the adaptation level.

Environmental stochasticity. At the end of each timestep the environment switches to a new state with probability $\varepsilon$. Simplifying we assume that while the state of the environment changes over time, the maximum adaptation levels for a given level of generality stays constant. We compared two different modes of environmental change that differed in the extent to which individuals could predict the new state of the environment after an environmental change. First a drastic change mode, where the environment can switch randomly to any new state in the interval $[-1,1]$, replicating very unpredictable environments (cf. solid line in Fig. 1b). Second a gradual change mode, where the environment changes according to $E(t+1)=E(t)+\delta$ with $\delta \sim$ $\mathcal{N}\left(0, \sigma_{\varepsilon}\right)$ (cf. dotted line in Fig. 1b). The variable $\sigma_{\varepsilon}$ controls the magnitude of the environmental change.

Simulation set-up. We ran 1000 independent simulations per analysis and parameter combination. Simulation runs were initialized by setting the state of the environment $E(t)$ to a random value in the interval $[-1,1]$. Each individual receives a starting variant with $\mu_{i}$ drawn from $\mathcal{U}(-1,1)$ and $\sigma_{i}$ drawn from $\mathcal{U}(0,2)$. We started recording variables of interest after a burn-in period of 1000 timesteps, which was long enough so that the system reached its equilibrium state. Consequently each simulation run started from a stochastic initial condition. Table 1 provides a summary of all parameters used in our model.

\section{Results}

Baseline model. We start our analysis by exploring a baseline model where all innovations are novel inventions and social learning occurs faithfully.

Adaptation dynamic after an environmental shock. The first set of simulations aims to uncover the adaptation dynamic after an environmental shock. In particular, we are interested in understanding how innovation rates and levels of generality of the present cultural variants respond. We assume a relatively stable environment $(\varepsilon=0.001)$ and after the burn-in period we let each simulation run until the next environmental change. We then record the mean adaptation levels, $a_{\text {pop }}(E(t))$ as well as the mean $\xi$-values, $\xi_{\text {pop }}(t)$, and $\sigma$-values, $\sigma_{\text {pop }}(t)$, of the population for additional $1.5 / \varepsilon$ timesteps (i.e. 1500 timesteps in relatively stable environments), where the environment stays constant. Figure 2 summarizes the result of 1000 simulation runs: the shaded areas show the $90 \%$ prediction intervals of these population-level quantities and the solid lines represent their corresponding mean values $\bar{a}_{\text {pop }}(E(t)), \quad \bar{\xi}_{\text {pop }}(t)$, and $\bar{\sigma}_{\text {pop }}(t)$ taken over the 1000 simulations.

If environmental conditions can fluctuate drastically (top four panels in Fig. 2), the adaptation process seems to consist of two phases. The first phase is marked by a sharp increase in both the mean level of generality, $\bar{\sigma}_{\text {pop }}(t)$, and the mean level of adaptation, $\bar{a}_{\text {pop }}(E(t))$, and the second phase by a decrease in $\bar{\sigma}_{\text {pop }}(t)$ and a moderate increase in $\bar{a}_{\text {pop }}(E(t))$. After a drastic environmental change it is likely that none of the existing variants are adapted anymore. In this situation generalist variants are more likely to provide some adaptive benefit, as their broader adaptation functions have a higher chance of covering the new state of the environment. Consequently, an increase in $\bar{\sigma}_{\text {pop }}(t)$ facilitates the quick discovery of variants with some adaptive benefit. However, as soon as most individuals have adopted these first successful variants, selection acts in the opposite direction as now more specialized variants may provide a higher level of adaptation leading to the decline in $\bar{\sigma}_{\text {pop }}(t)$. Comparing the top panels for $k=10$ and $k=1$ learners, it is obvious that a high number of learning opportunities results in a faster adaptation process.

If environmental conditions can fluctuate gradually (bottom four panels in Fig. 2 with $\sigma_{\varepsilon}=0.05$ ), qualitatively, a similar dynamic occurs but with a less pronounced first phase. After a gradual environmental change, some variants in the population, likely the ones with larger $\sigma$-values, may remain adapted to a certain extent and consequently only a relatively small increase in

\begin{tabular}{lll}
\multicolumn{2}{l}{ Table 1 Summary of model parameters. } \\
Parameter & Values & Description \\
\hline$E(t)$ & {$[-1,1]$} & State of the environment at time $t$ \\
$a_{i}(E(t))$ & {$[0,1]$} & Adaptation level of variant $i$ at time $t$ \\
$\mu_{i}$ & {$[-1,1]$} & Mean of the adaptation function of variant $i$ describing the environmental state to which it is best adapted to \\
$\sigma_{i}$ & $(0,0.2]$ & Standard deviation of the adaptation function of variant $i$ describing its level of generality \\
$a_{\max , i}$ & {$[0,1]$} & Maximum adaptation level of variant $i$ \\
$\xi_{j}$ & {$[0,1]$} & Probability of innovation for individual $j$ \\
$m$ & {$[0,1]$} & Probability of modification as opposed to novel invention \\
$\mu_{\xi}$ & 0.1 & Mutation rate for $\xi$ \\
$\varepsilon$ & $\{0.001,0.01,0.1\}$ & Probability the environment switches per time step \\
$\sigma_{\varepsilon}$ & $\{0.05,0.2\}$ & Standard deviation of the magnitude of gradual environmental change \\
$\sigma_{\xi}$ & $\{0.05,0.1,0.3\}$ & Standard deviation of the magnitude of gradual mutation on $\xi_{j}$ \\
$\sigma_{\text {error }}$ & $\{0,0.01,0.05\}$ & Standard deviation of the magnitude of copying error \\
$k$ & $\{1,3,5,10,20,50\}$ & Number of learning opportunities per timestep \\
\hline
\end{tabular}


the variants' level of generality are sufficient to track down the new state of the environment.

Notably, mean innovation rates, $\bar{\xi}_{\text {pop }}(t)$, (illustrated by the pink lines in Fig. 2) increase only very slightly at the beginning in the drastically fluctuating environment and even less in the gradually fluctuating environment in the case of 10 learners. This suggests that if individuals can modify their cultural variant frequently during their lifetime, there is little need for genetic evolution to modify learning parameters. In contrast, if only one individual can learn every timestep, i.e. individuals have fewer opportunities to modify their cultural variant during their life time, $\bar{\xi}_{\text {pop }}(t)$ also increases initially to facilitate the adaption process, especially after a drastic change in the environment. As expected, in the long term we observe a decrease in the innovation rates (with the lower limit determined by the mutation rate $\mu_{\xi}$ ).

A gradual mutation process of the parameter $\xi_{j}$, i.e. if it holds $\xi_{\text {offspring }}=\xi_{\text {parent }}+\delta$ with $\delta \sim \mathcal{N}\left(0, \sigma_{\xi}\right)$, leads to very similar dynamics when the magnitude of mutations, $\delta$, is relatively large (right panel in Fig. A2 in the Appendix). With small $\delta$-values (left panel), $\bar{\xi}_{\text {pop }}(t)$ increases only mildly after an environmental change, as the mutation process does not provide enough variation in the $\xi$-values for natural selection to act on. This inflexibility in the innovation rate is compensated by a more pronounced increase in $\bar{\sigma}_{\text {pop }}(t)$ compared to situations with larger $\delta$-values and therefore makes the flexible $\sigma$ strategy more important for the adaptation process.

We note that the adaptation dynamics are qualitatively similar in unstable environments (see Fig. A3 in the Appendix for $\varepsilon=0.01)$.

Interplay between innovation rate, trait characteristics, and learning opportunities. To further tease apart the interplay between innovation rate, level of generality, and learning opportunities, we ran a second set of simulations for different levels of environmental stability and different modes of environmental change and recorded relevant statistics after $t=1000$
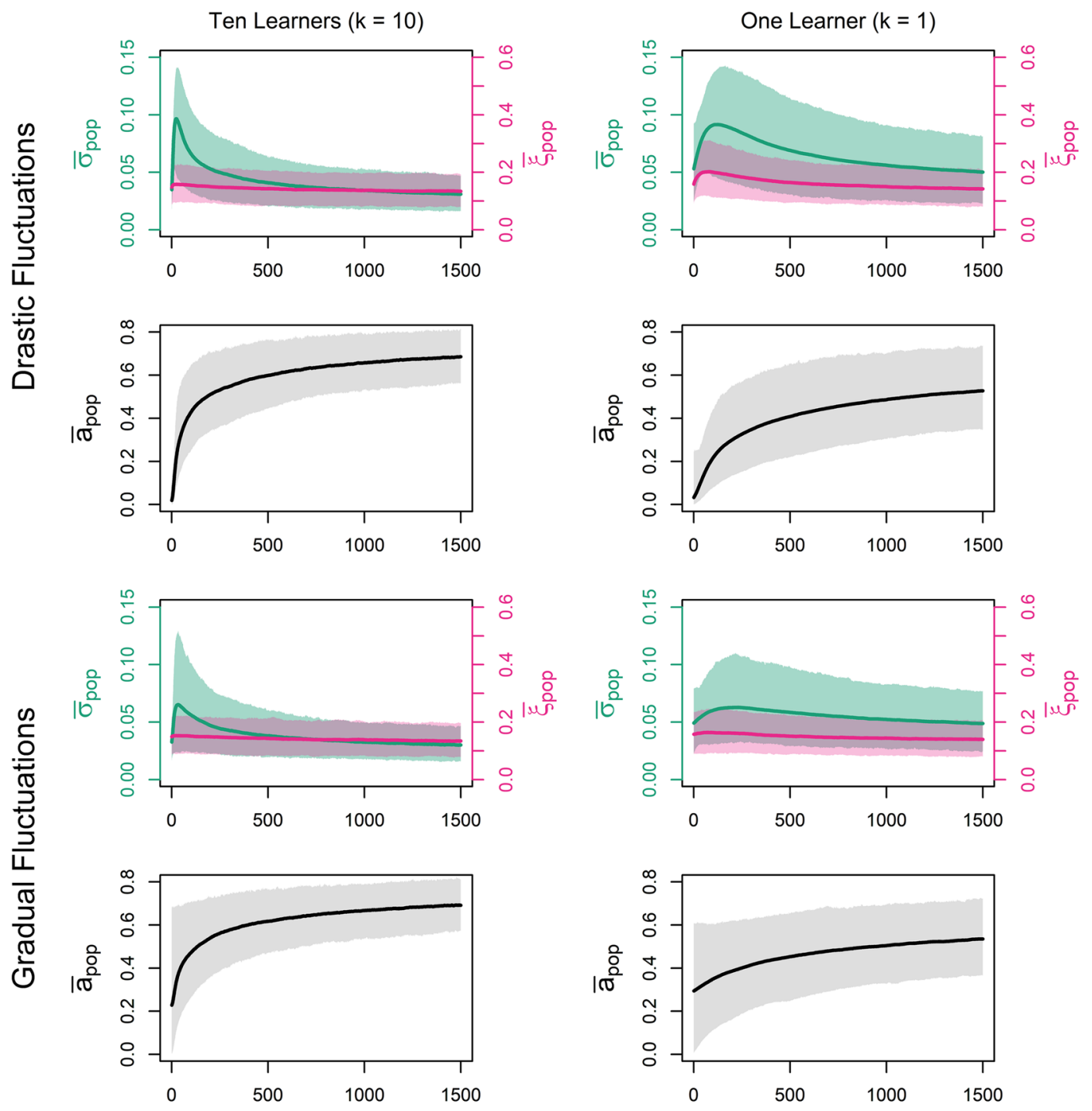

\section{Time since Environmental Change}

Fig. 2 Adaptation dynamics in relatively stable environments $(\varepsilon=0.001)$ for $k=10$ (left column) and $k=1$ (right column). Top panels show drastically changing environments, bottom panels gradually changing environments (with $\sigma_{\varepsilon}=0.05$ ). The solid pink lines illustrate the mean level of innovation, $\bar{\xi}_{\text {pop' }}$ in the population over time, the solid green lines show the mean level of generality, $\bar{\sigma}_{\text {pop }}$ and black lines show the mean adaptation level, $\bar{a}_{\text {pop }}$. The shaded areas represent the corresponding $90 \%$ Pls obtained from 1000 independent simulations. 
timesteps. Figure 3 plots the mean innovation rate, $\bar{\xi}_{\text {pop }}(t)$, against the mean level of generality, $\bar{\sigma}_{\text {pop }}(t)$, for different numbers of learning opportunities and different modes of environmental change.

For drastically changing environments (left panel in Fig. 3) we observe that higher rates of environmental change (illustrated by the different colors) result in more innovations and more generalist variants. The relative importance of both variables varies predictably with the number of learning opportunities: if individuals can update their cultural variant on average every other timestep (i.e. $k=50$ ), innovation rates remain relatively stable even for very different rates of environmental change. In other words, if the level of generality of the cultural variants is malleable enough, frequent adjustments of this variant characteristic suffice for successful adaptation to different rates of environmental change. With fewer learning opportunities, however, there is an increasing importance of innovations, or independent learning events, relative to the level of generality. In the extreme case of only one learner per timestep the mean innovation rate increases from under 0.2 in relatively stable environments (i.e. $\varepsilon=0.001$ ) to over 0.4 in relatively unstable environments (i.e. $\varepsilon=0.1$ ).

For "mild" gradual fluctuations (right panel in Fig. 3 with $\sigma_{\varepsilon}=0.05$ ), we find very similar innovation rates irrespective of the number of learning opportunities and environmental stability. Consequently, if environmental conditions change predictably within a certain range around the present state, individuals respond to more unstable environments (characterized by large $\mathcal{\varepsilon}$-values) by adopting more general variants not by innovating more. As expected, results for "strong" gradual fluctuations (middle panel in Fig. 3 with $\sigma_{\varepsilon}=0.2$ ) are intermediate between drastic environmental fluctuations and "mild" gradual fluctuations.

Summarizing, the relative contribution of the innovation rate and the level of generality of the cultural variants to the adaptation process in more unstable environments depends on the number of learning opportunities. While the innovation rate evolves through genetic processes, the level of generality evolves through cultural processes. The number of learning opportunities, $k$, effectively determines the relative timescales of both coevolutionary processes: if $k$ is large, cultural evolution is much faster than genetic evolution and individuals are more likely to find beneficial variants by increasing the variants' level of generality compared to genetically evolving higher innovation rates. In contrast, smaller values of $k$, make the "speed" of genetic and cultural evolution more similar resulting in a more significant role of frequent innovations in the adaptation process.

What good is a "flexible $\sigma$ " strategy? We have seen before that the mean level of generality, $\bar{\sigma}_{\text {pop }}(t)$ changes as part of the adaptation process. To better understand the functional significance of adjusting the variants' level of generality, we compare the "flexible $\sigma$ " scenario to scenarios where the variants' levels of generality were restricted to relatively high values ("generalists" with $\sigma_{i} \in[0.1,0.2]$ ) or relatively low values ("specialists" with $\left.\sigma_{i} \in(0,0.02]\right)$.

As before, after a burn-in period in a fluctuating environment $(\varepsilon=0.001)$, we let each simulation run until the next environmental change and record in Fig. 4 the proportion of 1000 independent simulations that reached a given adaptation threshold for different times after the environmental change: darker areas correspond to low proportions while lighter areas correspond to high proportions. As we are mostly interested in how adjusting the level of generality facilitates the adaptation to changing environments, we focus on the first phase of the adaptation process (see Fig. A4 in the Appendix for trajectories of 50 randomly selected simulation runs).

Populations with both fixed generalist (left panel in Fig. 4) and flexible variants (center panel in Fig. 4) found in all simulations variants with some positive adaptation level within the first few timesteps leading to a fast and reliable adaptation process. In contrast, populations with fixed specialist variants (right panel in Fig. 4) are characterized by a much higher variability in success. In some simulations the populations found quickly an adaptive solution while in others the populations remained not adapted even after 250 timesteps leading to a more unreliable adaptation process. Naturally, as time progresses the situation changes. Now populations with fixed generalist variants are restricted to rather low adaptation levels stagnating at around 0.3 , whereas populations with fixed specialist variants show much higher adaptation levels. Flexibly adjusting the variants' level of generality allows individuals to mitigate the trade-off between fast and reliable initial adaptation and the potential for long-term improvement if the environment stays unchanged. Increasing the $\sigma$-values of the variants in the beginning prevents the risk of long phases of nonadaptation, while reducing the $\sigma$-values again after the phase of
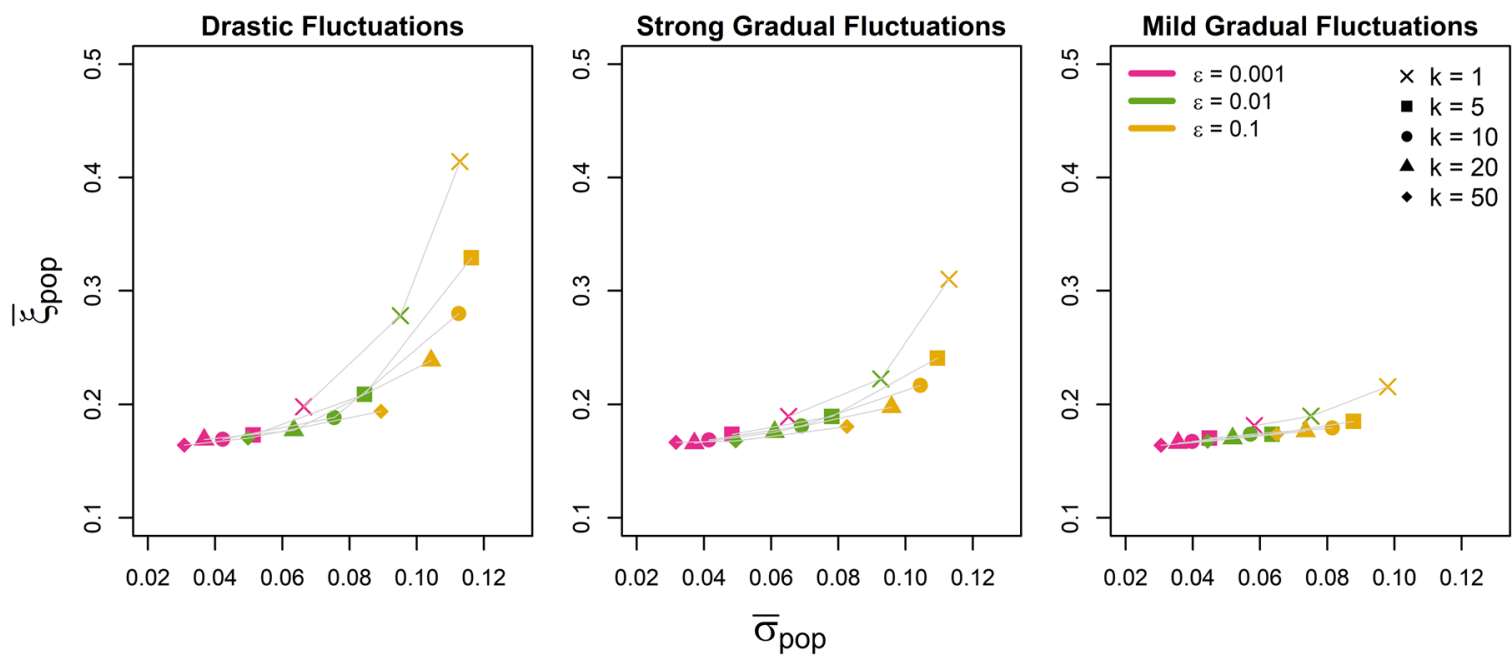

Fig. 3 Mean innovation rate, $\bar{\xi}_{\text {pop }}(t)$, vs. mean level of generality, $\bar{\sigma}_{\text {pop }}(t)$, for different rates of environmental change (indicated by color) and different numbers of learning opportunities (indicated by shape). Drastically fluctuating environments are shown on the left, strong gradual fluctuations $\left(\sigma_{\varepsilon}=0.2\right)$ in the middle and mild gradual fluctuations $\left(\sigma_{\varepsilon}=0.05\right)$ on the right. Gray lines connect simulations with same number of learning opportunities. 
initial adaptation allows individuals to improve adaptation levels and go beyond the levels fixed generalists can achieve.

Source of innovation. So far, we have assumed that individuals innovate only through novel invention. However, both theoretical (e.g. Lewis and Laland, 2012) and empirical (e.g. Miu et al., 2018) research suggests that individuals do not only invent new variants but heavily rely on gradual modification of their existing variants. To investigate how such gradual modification influences shortterm and long-term adaptation dynamics, we repeated the analyses presented in the previous section but included the possibility to modify one's trait instead of inventing a new one.

Figure $5 \mathrm{~d}$ shows the adaptation dynamic under the assumption that $80 \%$ of the innovation, or independent learning, events are modifications and $20 \%$ novel inventions (Fig. 5a shows result for independent inventions only). These values were chosen according to Miu et al. (2018), but in the Appendix we explore the effects of different fractions of modification and novel invention (see Fig. A5). First, including modification substantially reduced the variability in early success for fixed specialist variants and lead to faster and more reliable initial adaptation compared to situations with only novel inventions (cf. pink 90\% PI in Fig. $5 \mathrm{a}, \mathrm{d})$. Innovation through modification allows individuals to explore the adaptive qualities of variants close to their present variant, and therefore to carry out a "local" search, so that individuals with specialist variants are still likely to identify the present, unknown state of the environment. But in the long run adaptation dynamics for populations with fixed specialists and
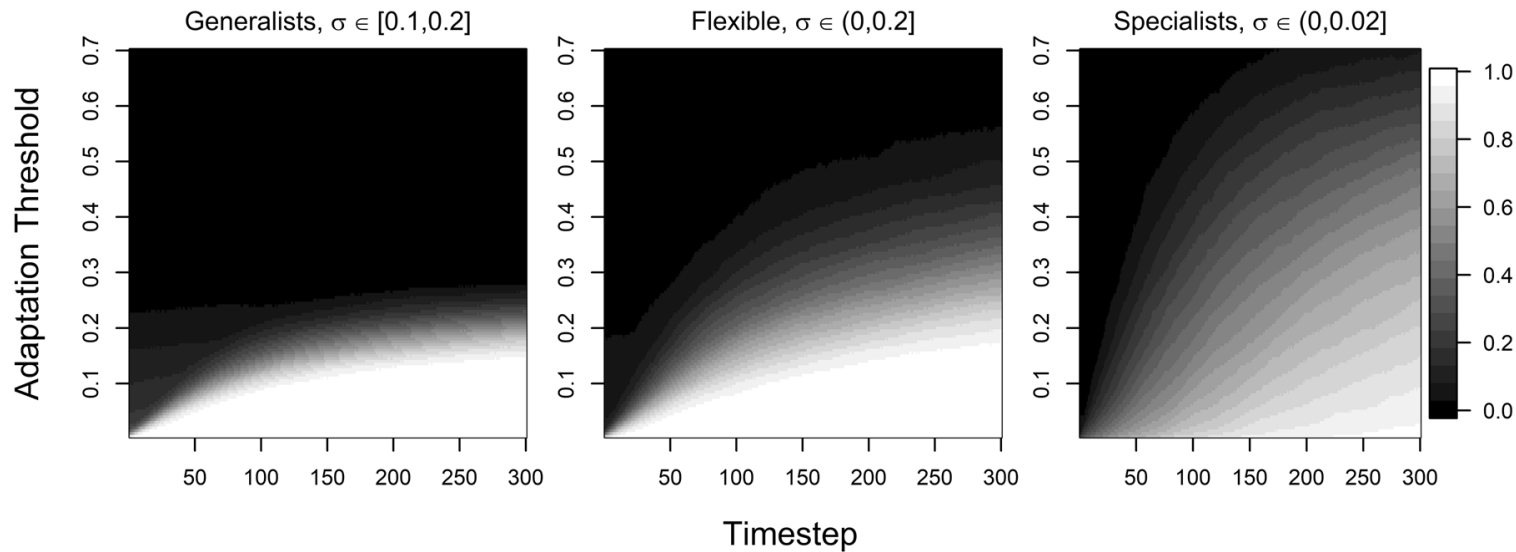

Timestep

Fig. 4 Proportion of simulations that reach a given adaptation threshold conditional on the time since the last change in the environment (with $k=1$ and $\varepsilon=0.001$ ). Results for generalist variants are shown in the left figure, for flexible variants in the middle figure, and for specialist variants in the right figure.
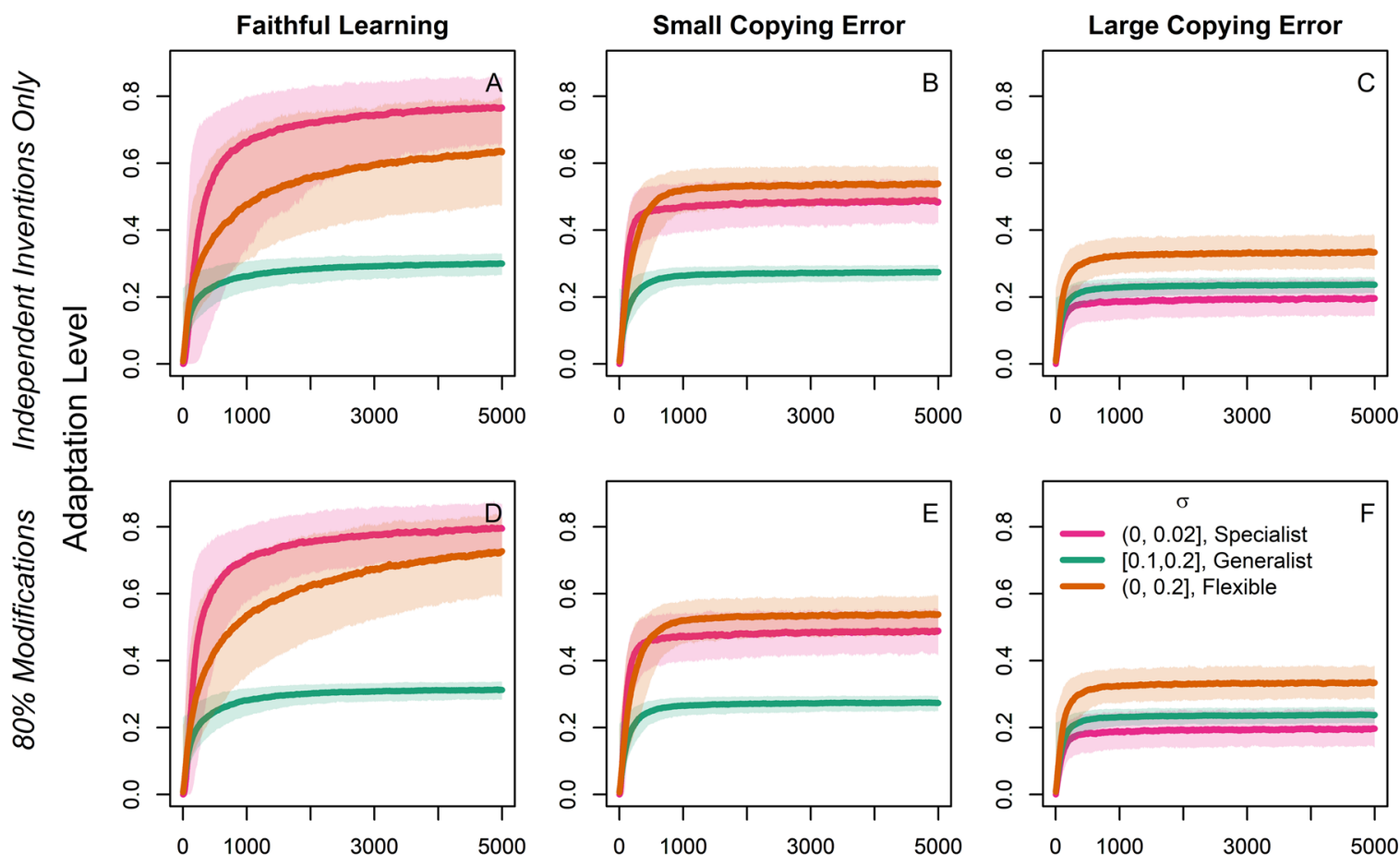

Time since Environmental Change

Fig. 5 Comparison of mean adaptation levels (and $90 \%$ PIs) of populations with $k=1$ for specialist variants $\left(\sigma_{i} \in(0,0.02)\right.$, solid pink lines and light pinkshaded areas), flexible variants $\left(\sigma_{i} \in(0,0.2]\right.$, solid yellow lines and light yellow-shaded areas) and generalist variants $\left(\sigma_{i} \in[0.1,0.2]\right.$, solid green lines and green-shaded areas). Top row shows results for novel inventions only, bottom row for $80 \%$ modifications and $20 \%$ novel inventions. Columns show results for faithful learning (left), small copying error $\left(\sigma_{\text {error }}=0.01\right.$; center) and large copying error $\left(\sigma_{\text {error }}=0.05\right.$; right). 
fixed generalists are very similar to situations with novel inventions. In contrast, modifications allow populations with variants with flexible levels of generality to reach a higher mean level of adaptation compared to situations of novel inventions only (cf. yellow solid lines in Fig. 5a, d). Once individuals have zoned in on the present state of the environment, "tinkering" with their variants allows them to more effectively reduce the $\sigma$-values and therefore to invent more specialized variants adapted to the present environmental state. Fig. A5 in the Appendix shows results for different fractions of modification and novel invention. While the described benefits generally increase with the proportion of modifications, there seems to be no further increase beyond $80 \%$ modifications. Notably, even $100 \%$ modifications lead to fast and reliable initial adaptation which might be attributable to the relatively easy search problem in this model.

Fidelity of social learning. Lastly, we relax the assumption of faithful social learning and include different levels of copying error. High-fidelity learning is often seen as a crucial prerequisite for cumulative cultural evolution, because it allows populations to maintain and build up adaptive cultural information over time (e.g. Lewis and Laland, 2012; Heyes, 1993; Tennie et al., 2009). In line with this reasoning, stronger reliance on low-fidelity social learning mechanisms, such as emulation in non-human primates, instead of imitation and teaching, is often seen as part of the explanation of why human culture is so exceptionally cumulative (Laland, 2018). However, high-fidelity social learning should only be adaptive if most models actually carry beneficial variants. Consequently, in fluctuating environments individuals may sometimes be better off if they do not faithfully copy what they observe but instead adopt a variant that is slightly different to the one they observed. To investigate this potentially twosided role of copying error, we run the same analyses as in the previous sections but with different levels of copying error. Figure 5 shows the adaptation dynamics for populations characterized by no copying error (left column), relatively small copying error (center column) and relatively large error (right column). Interestingly, even small copying errors allow populations (especially those with fixed specialist variants) to quickly and reliably adapt to new environmental conditions. Copying errors lead to slight changes to the variants individuals learn and consequently to an exploration of a wider range of environmental conditions. While modification results in the alteration of one's own variant, copying error results in the alteration of a variant copied from a model. The effects on short-term adaptation are comparable with copying errors leading to an even faster initial adaptation process. As expected from previous research, the presence of copying errors in the learning process reduces the mean level of adaptation in the long run. Why does modification increase long-term adaptation levels while copying error reduces it given their similar effect on short-term adaptation? Modification allows individuals to make small changes to their variants that get then passed on to the next generation allowing gradual accumulation. Copying error, in contrasts, makes such accumulation impossible as variants get changed in each transmission event. The strength of the influence of copying error, however, varies between different scenarios. While populations with generalist variants are only mildly affected by unfaithful social learning, populations with specialist variants almost quarter their maximum mean adaptation level compared to faithful social learning for $\sigma_{\text {error }}=0.05$. Notably, populations with flexible variants reach the highest adaptation levels for both levels of copying error suggesting that once social learning is unfaithful, it is particularly beneficial to adjust the variants' $\sigma$-values flexibly.

\section{Discussion}

Being optimally adapted to present environmental conditions is desirable, but individuals also need to be able to cope with changing environments. Here, we investigate mechanisms of cultural adaptation in changing environments and report a Moran-type evolutionary model where individuals could adjust (i) their propensity for innovation vs. social learning and (ii) the properties of the cultural variants they adopt, in particular the variants' level of generality, i.e. the range of environmental conditions in which they confer adaptive benefits.

We found that modifying the level of generality of cultural variants allows populations to navigate the trade-off between fast and reliable initial adaptation and the potential for optimal longterm improvements. After a change in the environment, more generalist variants allow individuals to quickly adjust to the new condition before more specialist variants let them become better and better adapted. Similarly, Kolodny et al. (2015) found that if environments change rarely a population's tool repertoire can become highly specialized, whereas more frequent changing environments lead to the accumulation of more generalist variants. Our results add on to those findings by explicitly demonstrating how general and specialist variants evolve. Further, our analysis sheds light on the varying importance of specific mechanisms of the adaptation process in different environmental and cultural settings.

Number of learning opportunities. The number of learning opportunities an individual has during its life time determined whether changes in the way individuals learn (i.e. increasing or decreasing innovation rates) or changes in a characteristic of the cultural variant itself (i.e. trait specialization) play a more dominant role in responding to changed environments. Standard models of cultural evolution predict that higher rates of environmental change lead to higher rates of innovation, or individual learning events (e.g. Boyd and Richerson, 1988; Rogers, 1988). If the environment changes rapidly, chances are high that the available social information is outdated, so that it is best to innovate, or learn individually. Our model replicates these findings in situations of low numbers of learning opportunities. But for high numbers of learning opportunities modifying the properties of the cultural variant itself, in particular broadening their adaptation function, appears more efficient to adapt to new environmental conditions than changing the way individuals learn. This suggests that if individuals can modify their cultural variant frequently during their lifetime, there is little need for genetic evolution to modify learning parameters. We note that this conclusion hinges on the assumption that individuals possess a genetically determined propensity for social learning, i.e. they can switch between individual and social learning but only at a given rate. But even if there are only a few learning opportunities in an individual's life time, the efficiency of adjusting the learning dynamics for the adaptation process depends on the way the environment is changing. While drastically changing environments often forced individuals to innovate more in order to find beneficial variants, gradually changing environments resulted in similar innovation rates irrespective of the frequency of environmental changes and individuals adapted by only adjusting the level of generality of their variants.

Mode of innovation. Typically, properties of individual learning are ignored in models of cultural evolution (Fogarty et al., 2015). Often these models assume that individual learners acquire the adaptive behavior with a certain probability (and often paying a learning $\operatorname{cost} c$ ), without specifying how such individual learning exactly works (McElreath and Boyd, 2008). Here we distinguished 
between two modes of innovation: modification and independent invention. We found that the long-term adaptation level in situations where individuals are able to modify the level of generality of cultural variants are influenced by the mode of innovation. Populations where $80 \%$ of the innovation events are modifications and $20 \%$ independent inventions reached a higher mean level of adaptation compared to populations relying on independent inventions only. Once individuals have zoned in on the present state of the environment, "tinkering" with their variants allows them to more effectively reduce the $\sigma$-values and therefore to invent more specialized variants adapted to the present environmental state. We note that we would expect larger differences in the adaptation dynamics of populations engaging in these different innovations modes at various degrees, if the present, unknown state of the environment becomes more difficult to identify. The validation of this point is left for future research.

Fidelity of social learning. Lastly, we investigated the role of learning fidelity for short-term and long-term adaptation dynamics. Social learners in our model used payoff information to identify individuals to learn from and in accordance with the previous cumulative culture literature, high-fidelity transmission was necessary to reach optimal levels of adaptation in stable environments in the long term. High copying errors do not allow variants to become highly specialized to the present environmental condition, which per definition results in a lower mean adaptation level. However, low-fidelity social learning facilitated rapid, short-term adaptation in changing environments. Every time individuals engage in social learning they effectively explore the adaptation level of a cultural variant similar to the one that was demonstrated and which consequently leads to a relatively fast identification of the new environmental state (at least in our relatively simple environment).

Unlike most models of cumulative culture, we studied the effect of innovation and transmission fidelity on adaptation levels, rather than the diversity or complexity of cultural variants. As humans mostly adapt behaviorally and culture is frequently regarded as key to our ecological success (Boyd et al., 2011; Henrich and McElreath, 2003), models of cumulative cultural evolution should not solely focus on the number of cultural variants or cultural complexity in a population, but also study the impact this cultural ratcheting has on adaptation to variable environments. Recombination of existing variants into new ones is frequently regarded as another important process in cumulative cultural evolution (Mesoudi and Thornton, 2018; Lewis and Laland, 2012; Miu et al., 2018). Simplifying, individuals possess in our model only one variant at a time and cannot combine variants to form new ones. In the future, these assumptions should be generalized to allow individuals to hold multiple variants and to include more realistic innovation processes.

Payoff-biased social learning functions similarly to selection in that better adapted variants increase in frequency after transmission events and is expected to be particularly beneficial as the probability of copying is directly related the benefit of the observed trait, rather than indirectly as, for example, for frequency-dependent and model-based biases (Boyd and Richerson, 1988). Future studies could extend our model and investigate different social learning strategies, such as conformity, prestige, or several other model-based biases (Kendal et al., 2018). Conformity, for example has been found to be positively correlated with innovation rate, such that strong conformity requires fewer conformists (Kandler and Laland, 2013). It would be elucidating to see if and how conformity might influence the dynamics of both innovation rate and trait specialization in our model.
In summary, the model presented in this paper suggests that allowing characteristics of cultural variants, such as the level of generality to flexibly respond to changing environmental conditions can enhance both short-term and long-term adaptation. Undoubtedly adjusting the way individuals learn is a viable strategy to adapt to changing environments, but our results show that especially in situations where individuals have several opportunities to change the cultural variant in their life time it may be more efficient to adjust what is learned than how it is learned. This points to the importance of incorporating a more detailed description of the properties of cultural variants into models of cultural evolution.

\section{Data availability}

This manuscript does not contain any empirical data. Simulation and plotting code necessary to reproduce all results and figures in the manuscript can be found on GitHub: https://github.com/ DominikDeffner/TraitSpecializationSocialLearning.

Received: 30 August 2019; Accepted: 6 November 2019; Published online: 26 November 2019

\section{References}

Aoki K, Feldman MW (2014) Evolution of learning strategies in temporally and spatially variable environments: a review of theory. Theor Popul Biol 91:3-19

Boyd R, Richerson PJ (1988) Culture and the evolutionary process. University of Chicago Press

Boyd R, Richerson PJ, Henrich J (2011) The cultural niche: why social learning is essential for human adaptation. Proc Natl Acad Sci USA 108(Suppl. 2): 10918-10925

Cavalli-Sforza LL, Feldman MW (1981) Cultural transmission and evolution: a quantitative approach. Princeton University Press

Fogarty L (2018) Cultural complexity and evolution in fluctuating environments. Philos Trans R Soc B 373(1743):20170063

Fogarty L, Creanza N (2017) The niche construction of cultural complexity: interactions between innovations, population size and the environment. Philos Trans R Soc B 372(1735):20160428

Fogarty L, Creanza N, Feldman MW (2015) Cultural evolutionary perspectives on creativity and human innovation. Trends Ecol Evol 30(12):736-754

Fogarty L, Wakano JY, Feldman MW, Aoki K (2017) The driving forces of cultural complexity. Hum Nat 28(1):39-52

Galef BG, Laland KN (2005) Social learning in animals: empirical studies and theoretical models. AIBS Bull 55(6):489-499

Henrich J, McElreath R (2003) The evolution of cultural evolution. Evol Anthropol 12(3):123-135

Heyes CM (1993) Imitation, culture and cognition. Anim Behav 46(5):999-1010

Heyes CM (1994) Social learning in animals: categories and mechanisms. Biol Rev 69(2):207-231

Hoppitt W, Laland KN (2013) Social learning: an introduction to mechanisms, methods, and models. Princeton University Press

Kandler A, Laland KN (2009) An investigation of the relationship between innovation and cultural diversity. Theor Popul Biol 76(1):59-67

Kandler A, Laland KN (2013) Tradeoffs between the strength of conformity and number of conformists in variable environments. J Theor Biol 332:191-202

Kendal RL, Boogert NJ, Rendell L, Laland KN, Webster M, Jones PL (2018) Social learning strategies: bridge-building between fields. Trends Cogn Sci

Kolodny O, Creanza N, Feldman MW (2015) Evolution in leaps: the punctuated accumulation and loss of cultural innovations. Proc Natl Acad Sci USA 112 (49):E6762-E6769

Laland KN (2018) Darwin's unfinished symphony: how culture made the human mind. Princeton University Press

Leadbeater E (2015) What evolves in the evolution of social learning? J Zool 295 (1):4-11

Lewis HM, Laland KN (2012) Transmission fidelity is the key to the build-up of cumulative culture. Philos Trans R Soc Lond B 367(1599):2171-2180

McElreath R, Boyd R (2008) Mathematical models of social evolution: a guide for the perplexed. University of Chicago Press

Mesoudi A (2011) Variable cultural acquisition costs constrain cumulative cultural evolution. PLoS ONE 6(3):e18239

Mesoudi A, Thornton A (2018) What is cumulative cultural evolution? Proc R Soc B 285(1880):20180712 
Miu E, Gulley N, Laland KN, Rendell L (2018) Innovation and cumulative culture through tweaks and leaps in online programming contests. Nat Commun 9:2321

Moran PAP (1958) Random processes in genetics. Math Proc Camb Philos Soc 54 (1):60-71

Rogers AR (1988) Does biology constrain culture? Am Anthropol 90(4):819-831

Slatkin M (1974) Hedging oneas evolutionary bets. Nature 250:704-705

Starrfelt J, Kokko H (2012) Bet-hedging--a triple trade-off between means, variances and correlations. Biol Rev 87(3):742-755

Tennie C, Call J, Tomasello M (2009) Ratcheting up the ratchet: on the evolution of cumulative culture. Philos Trans R Soc Lond B 364(1528):2405-2415

\section{Acknowledgements}

We thank Laurel Fogarty, Brendan Barrett, and members of the Department for Human Behavior, Ecology and Culture at the Max Planck Institute for Evolutionary Anthropology in Leipzig for constructive discussions and criticisms which helped in improving this paper.

\section{Competing interests}

The authors declare no competing interests.

\section{Additional information}

Supplementary information is available for this paper at https://doi.org/10.1057/s41599019-0360-4.
Correspondence and requests for materials should be addressed to D.D.

Reprints and permission information is available at http://www.nature.com/reprints

Publisher's note Springer Nature remains neutral with regard to jurisdictional claims in published maps and institutional affiliations.

cc (i) Open Access This article is licensed under a Creative Commons Attribution 4.0 International License, which permits use, sharing, adaptation, distribution and reproduction in any medium or format, as long as you give appropriate credit to the original author(s) and the source, provide a link to the Creative Commons license, and indicate if changes were made. The images or other third party material in this article are included in the article's Creative Commons license, unless indicated otherwise in a credit line to the material. If material is not included in the article's Creative Commons license and your intended use is not permitted by statutory regulation or exceeds the permitted use, you will need to obtain permission directly from the copyright holder. To view a copy of this license, visit http://creativecommons.org/ licenses/by/4.0/.

(C) The Author(s) 2019 